\title{
A dor pós-operatória como contribuinte do prejuízo na função pulmonar em pacientes submetidos à cirurgia cardíaca
}

\author{
Postoperative pain as a contributor to pulmonary function impairment in patients submitted to heart \\ surgery
}

Cristiane Mecca GIACOMAZZI ${ }^{1}$, Verlaine Balzan LAGNI², Mariane Borba MONTEIRO³

RBCCV 44205-846

\section{Resumo}

Objetivo: Avaliar a dor em pacientes submetidos à cirurgia cardíaca por esternotomia, verificando a localização e a intensidade da dor durante o período de internação. Também sua influência na função pulmonar e sua correlação com as características do indivíduo e do procedimento cirúrgico.

Método: A amostra foi composta de 30 indivíduos, nos quais foi avaliada a função pulmonar pré-operatória por espirometria e inspirometria de incentivo. Acompanharamse os pacientes no pós-operatório, por meio de protocolo com informações da cirurgia, função pulmonar e um protocolo de avaliação álgica (escala análoga visual e desenho do corpo humano). Utilizou-se estatística descritiva, o teste de MannWhitney e a correlação Spearman.

Resultados: A revascularização do miocárdio foi a cirurgia mais freqüente. A intensidade da dor no período de pósoperatório foi moderada e localizava-se inicialmente na esternotomia, persistindo até $05^{\circ}$ pós-operatório. $O$ volume inspiratório máximo teve relação significativa com a dor $(r=$ $-0,277 ; \mathbf{p}<0,05)$. Não se observou correlação significativa da dor com outras variáveis.

Conclusão: Observou-se prejuízo significativo da função pulmonar, não se restabelecendo completamente até o $5^{\circ}$ dia de pós-operatório. Apesar dos achados, a dor não se relacionou significativamente com as características dos indivíduos e do procedimento cirúrgico.

Descritores: Complicações pós-operatórias. Dor pósoperatória. Modalidades de fisioterapia. Procedimentos cirúrgicos cardíacos.

\footnotetext{
1 - Fisioterapeuta.

2 - Especialista em Cinesiologia; Fisioterapeuta do Hospital São Francisco.

3 - Mestre em Ciências Médicas; Professora do Centro Universitário IPA. Trabalho realizado no Centro Universitário Metodista de Porto Alegre (IPA). 


\section{Abstract}

Objective: To evaluate the pain in patients submitted to heart surgery using sternotomy, verifying the location and intensity of pain during the hospitalization period. A second aim was to evaluate its influence on the pulmonary function and its correlation with the characteristics of the individual and the surgical procedure.

Method: The series was composed of $\mathbf{3 0}$ individuals with the preoperative pulmonary function assessed by spirometry and incentive spirometry. Patients were followed in the postoperative period using a protocol with information on the surgery and the pulmonary function and a protocol for pain evaluation (visual analogue scale and body picture). Descriptivestatistics, Mann-Whitney test and Spearman correlation were used for statistical analysis.

\section{INTRODUÇÃO}

A dor é uma sensação freqüente após a cirurgia cardíaca. Mueller et al. [1] constataram que 51\% dos pacientes ainda apresentavam dor na região da esternotomia no $7^{\circ}$ dia após a cirurgia cardíaca. Também no estudo de Walther et al. [2], um número relativamente alto de pacientes no mesmo período ainda referia dor. Outros trabalhos sobre o tema mostram que a incidência de dor moderada chega a $43 \%$ e de dor grave a $34 \%$, dependendo da conduta do serviço $[3,4]$.

Apesar desses achados, entre 50\% e 75\% dos pacientes não recebem manejo analgésico adequado, levando ao risco de aumento da atividade simpática e eventos isquêmicos [3,5]. Também episódios de náuseas e vômitos, inibição dos movimentos e conseqüente aumento da chance de tromboembolismo são relatados [5], gerando prolongamento do tempo de hospitalização com conseqüentes custos que excedem a 28.000 Euros por paciente, em alguns países [6,7].

A dor originada de procedimentos de rotina do pósoperatório associada ao grande estímulo nociceptivo da esternotomia torna-se fonte importante de morbidade e mortalidade neste período [5,8], por influenciar a capacidade de tossir, respirar e movimentar-se adequadamente, podendo resultar em atelectasias (freqüentes em lobo inferior esquerdo, ocorrendo em até $90 \%$ dos pacientes) e pneumonias (29\%) [9,10].

A esternotomia mediana longitudinal foi realizada pela primeira vez em 1958 e é a incisão mais usada para as cirurgias cardíacas [11], sendo melhor para a exposição da região, porém pode alterar significativamente a função pulmonar pela conseqüente instabilidade do tórax superior [12,13]. O uso da artéria torácica interna esquerda (ATIE) também está associado com as alterações da função pulmonar porque este enxerto requer um trauma cirúrgico adicional, sendo mais doloroso para o paciente e implicando em uma clínica específica [1,12].
Results: Coronary artery bypass surgery was the most frequent surgery. The pain intensity in the postoperative period was moderate, initially located near to the sternotomy and persisted until the $5^{\text {th }}$ postoperative day. The maximum inspiratory volume had a significant relationship with pain $(r=-0.277 ; p<0.05)$. No significant correlation was observed between pain and others parameters.

Conclusion: Significant damage to the pulmonary function is observed which does not completely recover until the $5^{\text {th }}$ postoperative day. Despite these results, pain is not significantly associated to the characteristics of the individual or the surgical procedure.

Descriptors: Postoperative complications. Postoperative pain. Physical therapy modalities. Cardiac surgical procedures.

Apesar de todos os avanços, a dor no pós-operatório ainda parece estar subestimada. Muitos pacientes não solicitam analgesia, mesmo apresentando maiores intensidade de dor e outros recebem apenas $47 \%$ da dose efetiva do analgésico, segundo dados apresentados pelo estudo de Watt-Watson et al. [3]. Por meio da educação, pode-se tornar seu uso mais consciente e, em casos selecionados, utilizar a analgesia controlada pelo paciente de modo eficaz. Conhecer melhor as características da dor no pós-operatório e sua influência neste período é o início para o desenvolvimento de estratégias para melhorar seu controle.

A partir desses achados, realizamos este estudo com objetivo de avaliar a dor em pacientes adultos submetidos à cirurgia cardíaca por esternotomia, verificando a localização e a intensidade da dor durante o período de internação desses indivíduos. Também foi avaliada a sua influência na função pulmonar e sua correlação com as características do indivíduo e do procedimento cirúrgico.

\section{MÉTODO}

Este é um estudo de coorte, quantitativo, longitudinal e prospectivo, com amostra não-probabilística, sendo realizado entre março e dezembro de 2005, na Irmandade Santa Casa de Misericórdia de Porto Alegre (ISCMPA). Este trabalho foi aprovado pelos comitês de ética das instituições envolvidas, ambos em Porto Alegre (RS): Centro Universitário Metodista IPA (nº 1142, 28 de Setembro de 2004) e ISCMPA (n 930/04, 22 de Fevereiro de 2004).

Inicialmente, 120 pacientes foram selecionados para o estudo. Destes, 79 foram excluídos por não preencherem os critérios de inclusão: 51\% dos pacientes já foram submetidos a uma ou mais cirurgias cardíacas prévias, 17\% já realizaram outras cirurgias torácicas, 9\% dos indivíduos não realizaram esternotomia, 9\% dos pacientes recusaram-se a participar 
cardíaca

do estudo, 6\% apresentavam angina instável Classe III (classificação de Braunwald), 6\% foram operados em caráter de urgência ou emergência (impossibilitando colheita de dados pré-operatórios) e $2 \%$ dos indivíduos apresentavam câncer mediastinal.

Os pacientes foram incluídos neste estudo após entrevista para esclarecimento e assinatura do termo de consentimento livre e esclarecido. Participaram do estudo 41 pacientes, porém 11 deles não completaram todas as etapas e foram considerados perda do estudo (por retirada do consentimento, cirurgias adiadas para datas posteriores ao estudo ou cirurgia cancelada e óbito).

A amostra analisada foi composta de 30 indivíduos com idade de 53,9 \pm 13,93 anos (média \pm desvio padrão), de ambos os sexos, que foram submetidos, eletivamente, a cirurgia de revascularização do miocárdio (CRM) ou cirurgia valvar por esternotomia (com ou sem circulação extracorpórea - CEC) e que foram extubados de acordo com a rotina do serviço. Nenhum paciente apresentava doença pulmonar aguda no momento do estudo.

Foi realizada uma avaliação pré-operatória que consistia na colheita dos dados de identificação do paciente, informações detalhadas sobre fatores de risco e doenças associadas. Também constava a avaliação da função pulmonar por meio de espirometria e inspirometria de incentivo (para obtenção estimada da capacidade inspiratória máxima).

A função pulmonar (espirômetro da marca Micro Medical Limited ${ }^{\circledR}$ ) forneceu a capacidade vital forçada (CVF), o volume expiratório forçado no primeiro segundo $\left(\mathrm{VEF}_{1}\right)$, coeficiente expiratório forçado no primeiro segundo (VEF1/ CVF\%) e pico de fluxo expiratório (PFE). Nesta manobra, o paciente foi instruído a realizar uma expiração forçada a partir da capacidade pulmonar total. Para obtenção do volume inspiratório máximo (Vimáx), foi realizada a inspirometria de incentivo (aparelho da marca Voldyne ${ }^{\circledR}$ ), após 15 minutos de descanso. O paciente foi instruído a realizar uma inspiração lenta e profunda, expandindo o abdome, a partir da capacidade residual funcional. $\mathrm{O}$ inspirômetro de incentivo foi escolhido pelo baixo custo e fácil reprodutibilidade do método. Para ambos os testes, foram realizadas três manobras com um minuto de intervalo entre estas. Os pacientes foram avaliados na posição sentada no leito, estando a cabeceira entre $45^{\circ}$ e $90^{\circ}$. Foi evitada a posição sentada quando o indivíduo apresentou grande excesso de peso (evidenciado pelo índice de massa corpórea igual ou superior a $35 \mathrm{~kg} / \mathrm{m}^{2}$ ). Neste caso, reclinou-se a cabeceira a $30^{\circ}$. A espirometria seguiu os critérios estabelecidos pelas Diretrizes para Testes de Função Pulmonar [14]. O mais alto valor foi adotado, contanto que apresentasse diferença menor a $10 \%$ do segundo maior valor.

Os pacientes foram acompanhados no $1^{\circ}, 2^{\circ}, 3^{\circ}$ e $5^{\circ}$ dias após a cirurgia. O protocolo de acompanhamento consistiu em informações das características do procedimento cirúrgico e de dados da função pulmonar. Quando o paciente referiu dor, utilizou-se um protocolo específico para sua avaliação, antes das provas de função pulmonar. Este protocolo consistia em um desenho do corpo humano, com vista anterior e posterior, dividido em 37 áreas anatômicas. O paciente, então, foi instruído a marcar a área mais álgica. Juntamente, foi aplicada uma escala subjetiva análoga visual de 0 até 10 centímetros, com 0 representando nenhuma dor e 10 como a dor mais intensa para aquela área (protocolo de avaliação da dor utilizado por Mueller et al.[1]).

Todos os pacientes saíram do bloco cirúrgico com o dreno de tórax n³6 (marca Braile®), com localização variável, mas apenas cinco pacientes ainda estavam utilizando-o no início da colheita de dados. Assim, tornou-se difícil avaliar estatisticamente sua influência na dor.

No período de pós-operatório imediato, a analgesia utilizada pelo serviço consistia em uso de morfina (2 a 10 mg, lentamente, diluída em 9ml de água destilada acrescida de $1 \mathrm{ml}$ de morfina a cada 4 horas). A infusão foi contínua de 2 a $5 \mathrm{mg}$ por hora (quando necessário). A partir deste período, utilizou-se paracetamol 750mg (de escolha), quando necessário e paracetamol associado à codeína (4/4h). A dipirona ( $2 \mathrm{ml}$ ) foi usada quando necessário, diluída em $8 \mathrm{ml}$ de água destilada.

As avaliações do antes e depois da cirurgia foram realizadas de forma padronizada pelos autores.

A análise dos dados foi realizada por meio da estatística descritiva pela média e desvio-padrão. As variáveis contínuas sem distribuição simétrica foram apresentadas com mediana e percentis interquartis 25-75. O teste não paramétrico Mann-Whitney foi utilizado para relacionar as variáveis sem distribuição normal (características dos indivíduos) e a relação de Spearman para a análise da intensidade da relação entre as variáveis (função pulmonar e características do procedimento cirúrgico). Utilizou-se o nível de significância de $5 \%(p \leq 0,05)$. Os dados foram calculados pelo programa SPSS, versão 12.0 e 13.0.

\section{RESULTADOS}

Trinta pacientes participaram do estudo, sendo 73,3\% do sexo masculino, com fração de ejeção média de $61 \pm$ 13,9\%. A hipertensão arterial sistêmica (HAS) teve maior prevalência (76\%) entre as doenças associadas (seguida de dislipidemia com $28 \%$, diabetes mellitus e infarto agudo do miocárdio prévio, ambos com freqüência de 20\%). A doença pulmonar obstrutiva crônica (DPOC) também teve a maior prevalência entre as pneumopatias (16,7\%). Trinta por cento dos pacientes eram tabagistas, em média há 23,7 \pm 13,93 anos, consumindo cerca de 28,5 cigarros por dia. Nenhum 
dos pacientes havia sido submetido a cirurgia abdominal alta anteriormente e 36,7\% realizaram fisioterapia préoperatória. As características dos indivíduos e os principais fatores de risco para complicações pós-operatórias estão apresentados na Tabela 1.

Tabela 1. Características dos pacientes, fatores de risco para complicações pós-operatórias e características dos procedimentos cirúrgicos

\begin{tabular}{lccc}
\hline Variáveis & Total n=30 & Mínimo & Máximo \\
\hline Sexo masculino (n,\%) & $22(73,3 \%)$ & & \\
Idade (anos)* & $53,9 \pm 13,93$ & 17 & 78 \\
IMC (kg/m2)* & $25,65 \pm 3,95$ & 21,15 & 39,56 \\
FE (\%)* & $61,48 \pm 13,90$ & 32 & 82 \\
Tabagistas (n,\%) & $9(30 \%)$ & & \\
Hipertensão arterial & $19(76 \%)$ & & \\
Pneumopatias (n,\%) & & & \\
DPOC & $5(16,7 \%)$ & & \\
Asma & $2(2,7 \%)$ & & \\
Tempo de cirurgia (min)* & $297,4 \pm 66$ & 180 & 420 \\
CEC (n,\%) & $23(76,7 \%)$ & & \\
Tempo de CEC (min)* & $70 \pm 26,74$ & 27 & 123 \\
ASA & & & \\
3 & $16(88,9 \%)$ & \\
4 & $2(11,1 \%)$ & \\
Não disponível & 12 & \\
Tempo de intubação (horas)* & $13,72 \pm 4,3$ & 5,35 \\
\hline Abreviaçoes: IMC- & & \\
\hline
\end{tabular}

Abreviações: IMC- índice de massa corpórea; FE-fração de ejeção; DPOC- doença pulmonar obstrutiva crônica; CEC- circulação extracorpórea; ASA - American Society of Anesthesiologists, avaliação do estado físico. *valores de média \pm desvio-padrão.

Os procedimentos cirúrgicos realizados foram a revascularização miocárdica (CRM) (50\%), a troca valvar (49\%) e a ressecção valvar parcial (1\%). Quarenta por cento das CRM envolveram a ATIE, $10 \%$ envolveram a veia safena e $30 \%$ das válvulas substituídas foram metálicas. Na maioria dos pacientes (76,7\%) foi empregada CEC. As características dos procedimentos cirúrgicos são mostradas também na Tabela 1.

Os valores médios do $\mathrm{VEF}_{1}$ e da CVF no pré-operatório

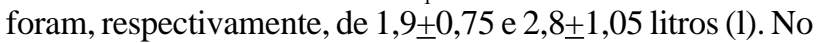
$1^{\circ}$ pós-operatório, os valores diminuíram significativamente para 0,66 $\pm 0,25$ e $1 \pm 0,36$ litros $(p<0,001)$ em relação ao préoperatório. $\mathrm{O} \mathrm{VEF}_{1}$ aumentou de modo significativo a partir $2^{\circ}$ pós-operatório $\left(0,83 \pm 0,34\right.$ litros, $\mathrm{p}<0,001$ e no $3^{\circ}$ pósoperatório $1,09 \pm 0,41$ litros; $\mathrm{p}<0,001$ ) até $05^{\circ}$ pós-operatório $1,23 \pm 0,58$ litros, $\mathrm{p}=0,047$. A CVF também aumentou diariamente de maneira significativa a partir do $2^{\circ}$ pósoperatório $\left(1,17 \pm 0,46\right.$ litros, no $2^{\circ}$ pós-operatório e 1,44_0,48 litros, no $3^{\circ}$ pós-operatório, ambos $\mathrm{p}=0,011$ ) até $\mathrm{o} 5^{\circ}$ pósoperatório $(1,71 \pm 0,62$ litros, $\mathrm{p}=0,008)$. A diferença estatística para essas variáveis ocorreu na comparação com o $1^{\circ}$ pósoperatório.

O valor médio de PFE no pré-operatório foi de 163+71,49 litros/minuto com queda significativa para 69,38 $\pm 25,21$ litros/ minuto, no $1^{\circ}$ pós-operatório $(\mathrm{p}<0,001)$. Os valores aumentaram diariamente de modo significativo na comparação com o $1^{\circ}$ pós-operatório. No $2^{\circ}$ pós-operatório, obteve-se $86,06 \pm 35,63$ litros/minuto e no $3^{\circ}$ pós-operatório 122,89+63,06 litros/minuto, ambos com $p=0,001$. No $5^{\circ}$ dia de pós-operatório, este valor atingiu 141,07 $\pm 76,71$ litros/ minuto $(\mathrm{p}=0,026)$ - Figura 1 . No pré-operatório, o Vimáx médio foi de 2266,6 $6 \pm 900,03$ mililitros, reduzindo significativamente para 646,15 $\pm 284,57$ mililitros no $1^{\circ}$ dia após a cirurgia $(\mathrm{p}<0,001)$. Assim como os outros parâmetros de função pulmonar, houve aumento significante no $2^{\circ}$ e $3^{\circ}$ dia após a cirurgia $(933,92 \pm 496,47$ mililitros e 1265,38 \pm 713,69, ambos $\mathrm{p}<0,001)$, com exceção do $5^{\circ}$ pós-operatório $(1425,96 \pm 732,27$ mililitros, $\mathrm{p}=0,089$ ) em relação ao $1^{\circ}$ pós-operatório.

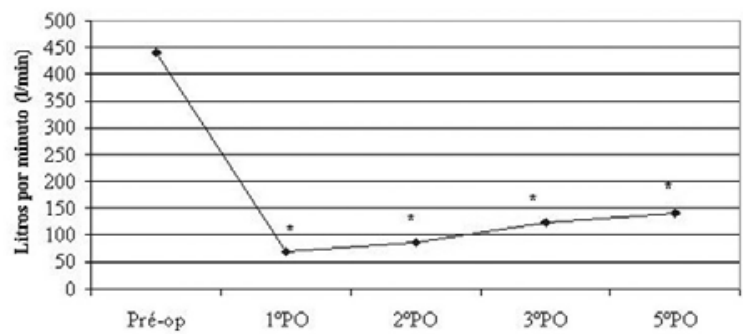

Fig. 1 - Valores médios diários de pico de fluxo expiratório. *p< 0,05 entre o pré e o $1^{\circ}$ dia de pós-operatório, entre $1^{\circ}$ e o $2^{\circ}$, entre $2^{\circ}$ e $3^{\circ}$ e entre o $3^{\circ}$ e $5^{\circ}$ pós-operatórios

Em relação à dor, apenas cinco pacientes não apresentaram este sintoma durante todo período de pós-operatório, no $1^{\circ}$ dia, 17 (56,6\%) pacientes referiram dor, no $2^{\circ}$ dia, 18 (60\%) indivíduos referiram este sintoma e no $3^{\circ}$ dia, apenas nove (30\%). No $5^{\circ}$ dia após a cirurgia, 11 (36,6\%) pacientes ainda referiam esta sensação. Dor de intensidade moderada $(6,17 \pm 3,04)$ foi referida no $1^{\circ}$ pós-operatório e esta intensidade diminuiu significativamente $(\mathrm{p}=0,003)$ no $2^{\circ}$ pósoperatório (3,95 $\pm 2,23$, dor de leve intensidade), porém voltou a subir no $3^{\circ}$ pós-operatório $(4,67 \pm 3,57, \mathrm{p}=0,859) \mathrm{e}$ permaneceu moderada até $05^{\circ}$ dia de pós-operatório $(4,7 \pm$ $3,60, \mathrm{p}=0,547)$. O local doloroso mais indicado pelos pacientes foi a região do esterno, exceto no $5^{\circ}$ dia após a cirurgia, sendo a região superior do membro inferior esquerdo 

cardíaca

(correspondendo à região próxima ao quadríceps) mais citada $(n=3 / 11)$ mesmo pelos pacientes que utilizaram a AITE.

Quando relacionada dor às características dos indivíduos, não houve significância entre a intensidade de dor e as variáveis sexo $(p=0,87)$, idade $(p=0,387)$ e IMC $(p=0,744)$. Também quando relacionadas às características da dor com as características cirúrgicas não foi observada significância com nenhuma variável, por exemplo, entre a intensidade de dor e o tempo de intubação $(p=0,248)$. O tipo de cirurgia também não se relacionou com a dor $(\mathrm{p}=0,970)$, assim como o tempo cirúrgico $(\mathrm{p}=0,812)$ e o tempo de CEC $(\mathrm{p}=0,07)$.

Tempo de analgesia não teve diferença estatística em nenhum dia de pós-operatório ( $\mathrm{p}=0,9$ para o $1^{\circ}$ e o $2^{\circ}$ pósoperatório, $\mathrm{p}=0,490$ para o $3^{\circ}$ pós-operatório e $\mathrm{p}=0,760$ para o $5^{\circ}$ pós-operatório). A relação uso de analgésico e intensidade de dor foi significativa no $1^{\circ}$ pós-operatório $(\mathrm{p}=$ $0,013)$, o que pode ser explicado pela elevada média de dor neste dia $(6,17 \pm 3,04)$.

Correlação próxima à significância, porém fraca, foi encontrada entre a intensidade de dor e o PFE $(r=-0,360$; $p=$ $0,051)$; e, embora significativa, a correlação entre a intensidade dor com o Vimáx também foi fraca $(\mathrm{r}=-0,277$; $\mathrm{p}=$ 0,04) - Figura 2.

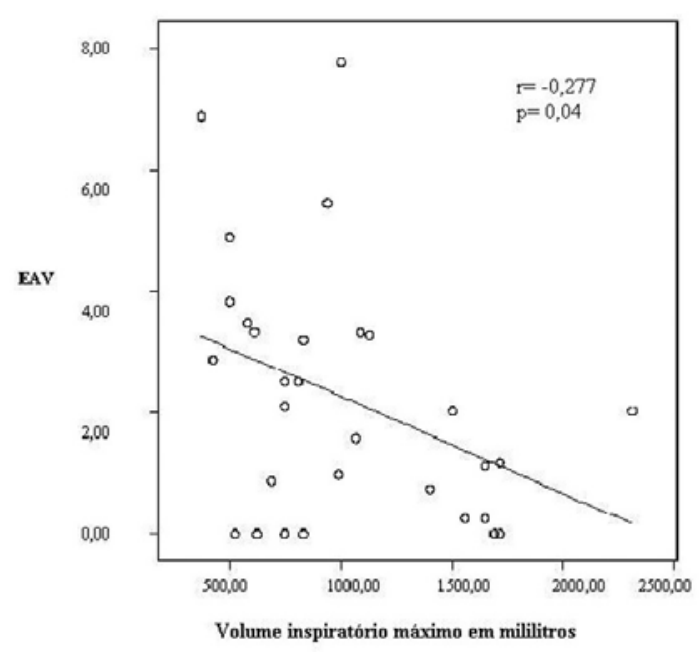

Fig. 2 - Relação entre intensidade de dor e volume inspiratório máximo, $r=-0,277 ; p=0,04$. EAV- escala análoga visual

\section{DISCUSSÃO}

No presente estudo, não observamos relações significantes entre as características dos indivíduos (idade, sexo e IMC, por exemplo) e do procedimento cirúrgico, como alguns autores sugerem, talvez pelo número pequeno da amostra. Mueller et al. [1] relatam que os pacientes com menos de 60 anos referiram maior intensidade de dor, com média de 4,3 $\pm 2,2$ (3,6 $\pm 2,4$ para pacientes com mais de 60 anos). As causas não são precisas, mas sugere-se que a possível dificuldade de comunicação dos idosos possa ser o motivo desses achados. Contrariamente, em outro trabalho do mesmo autor [15], pacientes idosos tiveram maior média de intensidade de dor, mas, neste estudo, o uso da ATIE teve prevalência em pacientes desta faixa etária. Da mesma forma, as razões para a diferença entre sexos masculino e feminino e a dor ainda são conflitantes. Indivíduos que apresentaram um IMC maior de $30 \mathrm{~kg} / \mathrm{m}^{2}$ também referiram mais dor, possivelmente pelo fato da dificuldade no manejo analgésico adequado destes [1]. Não foi possível realizar o cálculo estatístico dessa variável em nosso trabalho, pois somente três pacientes apresentaram o IMC maior de $30 \mathrm{~kg} /$ $\mathrm{m}^{2}$. No estudo de Mueller et al. [1], já citado, e de WattWatson et al. [3], o sexo feminino apresenta maior intensidade de dor. Neste último, o fato foi explicado pela menor freqüência de solicitação de analgésicos no período de pósoperatório pelos pacientes do sexo feminino. $\mathrm{O}$ fato de apenas oito mulheres participarem de nosso estudo também gera dificuldade para a avaliação desta variável.

Confirmando achados de Mueller et al. [1,15], a dor referida no presente estudo foi moderada (exceto pelo $2^{\circ}$ pós-operatório) e localizou-se na região da esternotomia até o $3^{\circ}$ pós-operatório, passando para o membro inferior associada à presença da safenectomia (no caso do estudo de Mueller, a dor passa a ter característica osteoarticular). Segundo os mesmos trabalhos, a maior intensidade de dor ocorreu no $2^{\circ}$ pós-operatório, no entanto, em nosso estudo, o $1^{\circ}$ pós-operatório apresentou maior intensidade de dor. Essa variação da localização e intensidade da dor pode ocorrer porque os estímulos nociceptivos iniciais diminuem com o passar do tempo (pela distância do procedimento cirúrgico, instabilidade esternal e uso de drenos, por exemplo) e como o paciente fica mais ativo no leito, sente outros aspectos relacionados à cirurgia como o prolongado posicionamento, efeitos de espasmos musculares relacionados ao procedimento e fratura de costelas [1,3,15]. Importante destacar também, em nossa pesquisa, que o $1^{\circ}$ dia de pós-operatório apresentou mais pacientes com dor que o $5^{\circ}$ dia (17 e 11 , respectivamente) e isso enfatiza a importância de uma analgesia inicial mais controlada.

Mueller et al. [1], em seu estudo, assim como em nosso, não encontraram diferença significativa nas características da dor quando comparados diferentes tipos de cirurgia, mesmo em procedimentos mais profundos, com maior estímulo nociceptivo como cirurgias valvares, mas ele não cita se foram incluídos pacientes que fizeram uso de ATIE. Porém, o mesmo autor, em outro trabalho [15], comparou pacientes que realizaram CRM usando ATIE com outros enxertos e constatou que a média de dor dos pacientes que 
recebem enxerto de ATIE é maior, provavelmente pelo trauma cirúrgico adicional que este enxerto impõe. Possivelmente, nesta pesquisa, essa relação poderia ser significante, com uma amostra de tamanho maior.

O prejuízo significante da função pulmonar no período de pós-operatório de cirurgia cardíaca é uma complicação bem conhecida, porém suas causas ainda estão sendo exploradas. Mueller et al. [15] afirmam que o impacto da AITE é moderado no pós-operatório, baseado no maior trauma cirúrgico que este enxerto exige (causando maior desconforto ao paciente), porém não realizam testes de função pulmonar. Já Çimen et al. [12] e Guizilini et al. [16] apontam a CEC como uma das causas para seu prejuízo devido ao aumento da resistência da via aérea e possível aumento de disfunção diafragmática, comparando a função pulmonar de pacientes que utilizaram ou não CEC. Ambos mostraram que há um prejuízo após a cirurgia cardíaca, porém, nos procedimentos envolvendo CEC, o dano é maior. O dreno de tórax também traz alteração significativa do $\mathrm{VEF}_{1}$ e da CVF no estudo de Guizilini et al. [17], sendo pior o dreno intercostal quando comparado ao dreno mediastinal, pela fricção provocada durante o movimento respiratório. Conseqüentemente, há irritação nervosa, causando maior sofrimento ao paciente. A própria incisão também é questionada por Walther et al. [2] e Lichtenberg et al. [13], mostrando que uma mini-incisão gera menor prejuízo à função pulmonar do que a esternotomia mediana, com menor intensidade de dor, tornando possível uma mobilização precoce, graças à estabilidade esternal. Porém. não podemos afirmar esta relação no presente estudo, pois os objetivos não abrangem este tema.

No presente trabalho, foi mostrada uma fraca correlação significativa entre a intensidade de dor e o Vimáx, confirmando estudos prévios sobre os danos da função pulmonar no pósoperatório de cirurgia cardíaca. Este achado confirma a alteração da função pulmonar também pela dor. A presença de dreno de tórax, principalmente intercostal, que perfura a pleura intercostal e músculos intercostais [17], limitando a inspiração, é uma causa. Em nosso estudo, poucos pacientes utilizavam dreno de tórax no momento da colheita de dados, por isso, é muito difícil sugerir o uso de dreno como contribuição para diminuição significativa na Vimáx nos dados apresentados. Também o uso da AITE, apesar do nosso estudo não ter encontrado relação entre essas variáveis, pode influenciar a capacidade inspiratória. Isso por representar um trauma cirúrgico adicional e diminuir o aporte sanguíneo para a musculatura intercostal, reduzindo a força muscular ventilatória [15,18]. Outra possibilidade é a disfunção diafragmática causada pela própria manipulação cirúrgica, que determina inibição do nervo frênico (possivelmente pela cardioplegia) e paresia diafragmática $[5,16]$. Entretanto, este tema específico necessita de maiores investigações.
A cirurgia cardíaca é um procedimento que causa agressão ao organismo, também pela esternotomia. Apesar de não ter sido avaliada a influência da esternotomia na dor, esta poderia ser atribuída à extensão da incisão [4,13] e fricção do esterno pela instabilidade do tórax superior $[2,16]$. Os baixos valores espirométricos, juntamente com os baixos e significativos valores de capacidade inspiratória máxima também podem refletir o medo ou o desinteresse do paciente em colaborar com os testes de função pulmonar.

Com um adequado manejo analgésico, a dor e conseqüentemente a função pulmonar pós-operatória podem melhorar [5,15]. Fato importante principalmente em idosos, que já têm sua capacidade pulmonar diminuída (com maior risco de desenvolver complicações pulmonares) [19] e que com os avanços representam um número crescente de pacientes submetidos a procedimentos cirúrgicos.

Em nosso estudo, não realizamos uma avaliação radiológica, que poderia evidenciar complicações pulmonares de diferentes proporções, afetando os valores de função pulmonar. Também o protocolo analgésico utilizado pela instituição onde foi realizada a pesquisa é diferente de alguns trabalhos encontrados sobre este assunto, que utilizam analgesia epidural, sendo assim de difícil comparação com os nossos resultados. Os testes de função pulmonar representam uma limitação, como já citado, pois seus resultados dependem da compreensão das manobras e da vontade do pacientes em realizá-las. A metodologia para avaliação da capacidade inspiratória máxima não é mais específica, porém de fácil reprodutibilidade e baixo custo.

Trabalhos prévios sugerem que pacientes mais preparados e colaborativos com a equipe de reabilitação possam identificar precocemente a dor [18]. Como a dor é um sintoma freqüente no pós-operatório de cirurgia cardíaca, os profissionais da equipe de reabilitação também devem receber educação sobre este tema, visando a sua identificação e manejo precoce.

\section{CONCLUSÃO}

No presente estudo, a dor inicialmente localizou-se na região da esternotomia, tendo intensidade moderada. Porém, ela não se correlacionou com as características dos indivíduos e do procedimento cirúrgico, possivelmente relacionado ao tamanho da amostra, onde algumas variáveis têm freqüência muito pequena para a realização do cálculo estatístico. Ainda assim, observou-se o prejuízo significativo da função pulmonar, que não se restabelece até o $5^{\circ}$ dia de pós-operatório. Essa alteração pode ser melhor percebida com a relação significativa da dor com o Vimáx. Também a dor foi uma queixa que persistiu durante todo período do estudo. 


\section{REFERÊNCIAS}

1. Mueller XM, Tinguely F, Tevaearai HT, Revelly JP, Chiolero $\mathrm{R}$, Segesser LK. Pain location, distribution, and intensity after cardiac surgery. Chest. 2000;118(2):391-6.

2. Walther T, Falk V, Metz S, Diegeler A, Battellini AR, Autschbach R, et al. Pain and quality of life after minimally invasive versus conventional cardiac surgery. Ann Thorac Surg. 1999;67(6):1643-7.

3. Watt-Watson J, Stevens B, Katz J, Costello J, Reid GJ, David T. Impact of preoperative education on pain outcomes after coronary artery bypass graft surgery. Pain. 2004;109(1-2):73-85.

4. Power I. Recent advances in postoperative pain therapy. Br J Anaesth. 2005;95(1):43-51.

5. Magnano D, Montalbano R, Lamarra M, Ferri F, Lorini L, Clarizia S. Ineffectiveness of local wound anesthesia to reduce postoperative pain after median sternotomy. J Card Surg. 2005;20(4):314-8.

6. Reimer-Kent J. From theory to practice: preventing pain after cardiac surgery. Am J Crit Care. 2003;12(2):136-43.

7. Pasquina P, Tramer MR, Walder B. Prophylactic respiratory physiotherapy after cardiac surgery: systematic review. BMJ. 2003;327(7428):1379-85.

8. Mueller XM, Tinguely F, Tevaearai HT, Ravussin P, Stumpe $\mathrm{F}$, von Segesser LK. Impact of duration of chest tube drainage on pain after cardiac surgery. Eur J Cardiothorac Surg. 2000;18(5):570-4.

9. Abboud C. Infecção em pós-operatório de cirurgia cardíaca. Rev Soc Cardiol Estado São Paulo. 2001;5:915-21.

10. Johnson D, Kelm C, Thomson D, Burbridge B, Mayers I. The effect of physical therapy on respiratory complications following cardiac valve surgery. Chest. 1996;109(3):638-44.
11. Anger J, Farsky PS, Amato VL, Abboud CS, Almeida AF, Arnoni RT, et al.. A utilização de retalho composto de pele e tecido mamário na reparação da área cruenta resultante da deiscência de esternotomia em cirurgia cardíaca. Arq Bras Cardiol. 2004;83:43-5.

12. Çimen S, Özkul V, Ketenci B, Yurtseven N, Günay R, Ketenci $\mathrm{B}$, et al. Daily comparison of respiratory functions between on-pump and off-pump patients undergoing CABG. Eur J Cardiothorac Surg. 2003;23(4):589-94.

13. Lichtenberg A, Hagl C, Harringer W, Klima U, Haverich A. Effects of minimal invasive coronary artery bypass on pulmonary function and postoperative pain. Ann Thorac Surg. 2000;70(2):461-5

14. Pereira CAC, Jansen JM, Barreto SSM, Marinho J, Sulmonett N, Dias RM. Espirometria. In: Diretrizes para testes de função pulmonar. J Pneumol. 2002;28(Supl 3):S1-S82.

15. Mueller XM, Tinguely F, Tevaearai HT, Revelly J, Chioléro $\mathrm{R}$, Segesser L. Pain pattern and left internal mammary artery grafting. Ann Thorac Surg. 2000;70(6):2045-9.

16. Guizilini S, Gomes WJ, Faresin SM, Bolzan DW, Alves FA, Catani R, et al. Avaliação da função pulmonar em pacientes submetidos à cirurgia de revascularização do miocárdio com e sem circulação extracorpórea. Rev Bras Cir Cardiovasc. 2005;20(3):310-6.

17. Guizilini S, Gomes WJ, Faresin SM, Carvalho ACC, Jaramillo JI, Alves FA, et al. Efeitos do local de inserção do dreno pleural na função pulmonar no pós-operatório de cirurgia revascularização do miocárdio. Rev Bras Cir Cardiovasc. 2004;19(1):47-54.

18. Leguisamo CP, Kalil AKR, Furlani AP. A efetividade de uma proposta fisioterapêutica pré-operatória para cirurgia de revascularização do miocárdio. Rev Bras Cir Cardiovasc. 2005;20(2):134-41.

19. Vasconcelos Filho PO, Carmona MJC, Auler Junior JOCA. Peculiaridades no pós-operatório de cirurgia cardíaca no paciente idoso. Rev Bras Anestesiol. 2004;54(5):707-7. 